\title{
Feminización de la migración ecuatoriana
}

\author{
Editado por/Edited by: Victor Cabezas \\ Recibido/Received: 9/11/2014. Aceptado/Accepted: 16/05/2015 \\ Publicado en línea/Published on Web: 16/10/2015
}

\author{
Kiara Guerra \\ Universidad San Francisco de Quito, Colegio de Ciencias Sociales y Humanidades COCISOH \\ Diego de Robles y Vía Interoceánica, Quito, Ecuador
}

Correo Electrónico: guerra_ki@yahoo.com

\section{Resumen}

La feminización de la migración es un proceso de movilidad humana que responde a muchas situaciones sociales, económicas e incluso políticas que han tenido lugar a nivel mundial. Este artículo trata sobre el caso particular del Ecuador, donde la ola migratoria femenina principalmente hacia Europa fue consecuencia de una época crítica que atravesó el país a finales de los años noventa. A continuación, exploramos las circunstancias que produjeron dicho fenómeno migratorio; también analizamos los roles de género de hombres y mujeres en los países de origen y de destino y la maternidad transnacional.

Palabras clave: feminización, migración, maternidad transnacional, plazas laborales, roles de género.

\section{Abstract}

The feminization of migration is a human mobility process that responds to several social, economic and even political situations that have taken place worldwide. Regarding the case of Ecuador female migration wave to Europe was primarily the result of a critical period that the country faced in the late nineties. The article explores the circumstances that produced such migration; we also analyze gender roles of men and women in their country of origin and destination as well as transnational motherhood.

Keywords: feminization, migration, transnational motherhood, work places, gender roles

$\int$ a investigación liderada por académicos en el ámbito de la movilidad humana a nivel mundial, así como muchas otras áreas de estudio, se ha desarrollado con un sesgo androcéntrico, es decir, que generalmente se toma como referencia de estudio sólo a los varones. Sin embargo, en los últimos años hemos sido testigos de la feminización de varios procesos sociales, tales como la pobreza y la migración, lo que ha impulsado a varios expertos a orientar sus estudios en estas dinámicas desde otra perspectiva, una que parte de la cantidad de mujeres que deciden encabezar procesos migratorios. De acuerdo con las cifras del Instituto Nacional de Estadística (INE) de 2004, sólo en 1998 migraron a España un aproximado de 3972 ecuatorianos, de los cuales 2346 eran mujeres (Torres, 2004). Teniendo en cuenta esta evidencia, las investigaciones sobre este tipo de movilidad humana empezaron a enfocarse en los cambios sociales relacionados con las dinámicas de migración en los países de origen y de destino, como consecuencia de un aumento en el flujo de mujeres que encabezaban planes migratorios hacia países desarrollados, es decir, como consecuencia de la feminización de la migración. A continuación, una revisión sobre cuándo y cómo se dio este proceso de movilidad en el Ecuador y también una reflexión sobre dos aspectos fundamentales, por un lado el tipo de trabajo que las mujeres migrantes realizan en los países de destino, y por otro los distintos cambios en las dinámicas de roles de género y de negociación dentro de la familia migrante.

\section{Antecedentes}

El contexto global en el que se evidencia la evolución de la feminización de la migración, se en- 
tiende en definitiva como parte del capitalismo postfordista en el que vivimos. Vale la pena recalcar que el postfordismo es una teoría que hace referencia a un sistema económico de producción, consumo y de fenómenos socioeconómicos y que supone tiene lugar en la actualidad. Éste se caracteriza, por ejemplo, por la feminización de la fuerza de trabajo, el aumento en la demanda de trabajadores no manuales, entre otros (Britannica).

De esta manera, el considerable aumento en el número de mujeres migrantes de Latinoamérica hacia Europa y Estados Unidos, se debió a cambios sustanciales en los mercados laborales globales, en particular el aumento en la demanda de fuerza laboral femenina de países en vías de desarrollo, de modo que se pudieran llenar los crecientes nichos laborales en el sector del cuidado y el de servicio doméstico de países desarrollados, debido al envejecimiento de la población y al aumento en la tasa de participación de la mujer en el mercado laboral profesional. A lo largo de este artículo al mencionar 'sector del cuidado' hacemos referencia a todos los trabajos que involucren a una persona cuidando de otra, por ejemplo, niñeras, guarderías o geriátricos. Por ejemplo, en Génova del total de ecuatorianos registrados, el $62.2 \%$ eran mujeres, frente a un $37.8 \%$ de hombres migrantes.

Adicionalmente, a lo largo del periodo de regularización en 2002, 25.009 demandas fueron asignadas a plazas laborales relacionadas con los servicios del cuidado frente a 10.829 demandas para trabajadores en otras áreas (Genta, N., \& Ramírez, J., 2008). Por lo tanto, la crisis en el sector del cuidado de los países europeos y de Estados Unidos, sumado a las crisis económicas, sociales y políticas de países como Ecuador, resultó en el planteamiento a nivel nacional de nuevas estrategias de supervivencia de las familias más afectadas por dichas condiciones en sus países de origen, es entonces cuando se inicia la migración de mujeres que puedan llenar los nichos laborales del sector del cuidado y del doméstico en los países de destino, mientras sustentan a sus familias en sus países de origen (Penson, 2007).

La feminización de la migración latinoamericana ha tenido distintas épocas en cada país, por ejemplo, la primera fuerte migración femenina latinoamericana salió de República Dominicana en los años sesenta (Torres, 2004). En el caso particular del
Ecuador, la feminización de la migración se dio entre 1998 y 2001, aproximadamente. Durante este periodo se estima que la mayor parte de las emigrantes salieron de provincias de la sierra sur de nuestro país, tales como Cañar, Loja y Azuay las cuales tienen una historia de altos flujos migratorios al exterior desde los años setenta. El destino principal de todas las mujeres que durante este periodo encabezaron los proyectos migratorios familiares fue Europa, particularmente a España, Italia y Bélgica (Pedone, 2010). Cabe recordar, que esta ola migratoria se da en un contexto histórico muy particular en el Ecuador; pues existía un ambiente de inestabilidad política, se vivía una crisis social y económica muy fuerte, aumentó el desempleo y el subempleo, así como también la pobreza. En 1999-2000 el Ecuador vivió una crisis económico-financiera, el hundimiento del aparato productivo nacional, y la pérdida de competitividad a nivel internacional causado por la dolarización. Por otro lado, en Europa, en lo referente al sector del cuidado y el sector doméstico, aumentó la búsqueda de mujeres, para su incorporación en este mercado laboral. Todos estos factores impulsaron, en la mayor parte de casos, a que las familias tomaran la decisión de migrar. La diferencia fundamental entre la migración masiva de 1998-2001, con anteriores, no es únicamente el país de destino, sino también los nichos laborales disponibles y por ende quién encabezaría finalmente el proyecto migratorio familiar.

Una vez que las familias ecuatorianas se ven obligadas a migrar, a causa de la crisis nacional en el Ecuador, deben decidir su destino. La mayor parte de ellas opta por migrar hacia Europa, que les ofrece ciertas garantías físicas que, Estados Unidos, en especial la travesía al Norte, no garantiza. A su vez, resulta más barato que emprender un viaje con los conocidos coyoteros (Pedone, 2010). Realizar el viaje a España, Italia o Bélgica era más sencillo, ya que no implicaba cruzar a pie una frontera; sin embargo, una vez en el país de destino, las situaciones a las que deberán enfrentarse no son menos complicadas que aquellas con las que habrían tenido que lidiar en América del Norte (Pedone, 2010). Las mujeres ecuatorianas encuentran en los países de europeos, un escenario no esperado, ya que este periodo migratorio femenino hacia España, coincide con un cambio en las leyes en este lugar en particular, como resultado existen menos derechos y más restricciones para las mujeres migrantes (Pedone, 2010). 


\section{Inserción laboral}

Una vez que las mujeres ecuatorianas llegan a los países de destino, buscan entrar en aquellas plazas laborales relacionadas con el cuidado y el servicio doméstico. Aquellos, han tenido una creciente demanda de mano de obra femenina, en virtud de que "el sistema económico globalizado, con una lógica patriarcal perversa, ubica a las migrantes en los puestos de trabajo más invisibles, vulnerables y mal remunerados, reproduciendo en el ámbito laboral los roles de servicio asignados tradicionalmente a las mujeres en todas las sociedades" (Romero, 2011). Es decir, que si bien son las ecuatorianas quienes comienzan a guiar la migración familiar, ellas en muchos casos pasarán a cumplir los mismos trabajos relacionados con el sector del cuidado y del servicio doméstico que realizaban en sus países de origen. Es así que, tomando como referencia la categoría de empleada doméstica, el 5\% de las mujeres ecuatorianas migrantes a Estados Unidos y Europa lo era antes de emigrar, y el 32\% lo es ahora en su país de destino; por ejemplo, en España alcanzan el 38\%, mientras que en Italia suman el 63.6\% (Genta, N., \& Ramírez, J., 2008).

Por ende, es evidente que la migración femenina no representa un cambio fundamental en la concepción general de cuáles son los trabajos destinados a las mujeres, pues hemos visto que en muchos casos éstos se mantienen, e incluso llama la atención que quienes no desempeñaban labores vinculados a esos ámbitos de trabajo terminarán haciéndolo tras la migración. Cabe aclarar que el objetivo de estos estudios no es deslegitimar dichas plazas laborales, ni mucho menos el arduo trabajo que muchas mujeres y hombres realizan en el ámbito del cuidado y del servicio doméstico; el objetivo principal es resaltar el hecho, de que la migración no supone en este aspecto, un cambio positivo para las migrantes ecuatorianas, si no que termina por encasillarlas en los mismos roles de género en los que muchas de ellas vivían en su país de origen.

\section{Roles de género y maternidad transnacional}

Los roles de género, aquellas prácticas que son legitimadas en la sociedad a través de mecanismos de poder y de dominación que son parte de la reproducción social y cultural, así como la socialización de género, es decir, la manera por la cual los diferentes espacios sociales configuran la subjetividad del ser mujer y ser hombre en la sociedad, sufren transformaciones como resultado de procesos sociales complejos. Los cambios en las dinámicas del mercado laboral y de la migración, por ejemplo, extraen a la mujer de su espacio habitual y la colocan en uno muy distinto, lo que implica cambios y transformaciones en los roles y la socialización de género, los cuales pueden favorecerla o no en la nueva sociedad de la que pasa a formar parte.

Estos cambios son evidentes, no tanto en el tipo de actividades que las mujeres migrantes realizarán en destino, sino fundamentalmente por el hecho de ser quienes encabezan la migración y por el papel protagónico que adquieren como resultado en las siguientes fases del ciclo y del proyecto migratorio familiar. Por ejemplo, cuando la mujer se convierte en el primer eslabón de una cadena migratoria, ella se transforma en el actor reagrupante del resto de la familia en el país de destino, es decir, que buscará la manera de trasladar progresivamente a todos los miembros de la familia al nuevo país de residencia. En las primeras olas migratorias ecuatorianas, el varón era quien migraba, y la mujer quien quedaba al cuidado del resto de la familia. En estos casos, la mujer se convertía en una figura de estabilidad emocional para su compañero, y por lo tanto, el momento en que la mujer migraba al mismo destino que su esposo, se simboliza la estabilidad emocional y la reintegración familiar.

Los roles de hombres y mujeres en nuestra sociedad han sido definidos, y aún lo siguen siendo, por instituciones de poder como el Estado, las iglesias y los procesos de difusión y socialización religiosa, el sistema educativo e incluso los medios de comunicación masivos, quienes se han encargado de construir y configurar los roles masculino y femenino (Pedone, 2010). Como resultado, han logrado cristalizar en nuestra sociedad el estereotipo de la mujer como madre, como la encargada de asegurar la reproducción, de transmitir los valores culturales y de mantener la red de cuidado de niños y ancianos, mientras que el padre corresponde al estereotipo del varón, el cual se concentra en la figura de autoridad dentro del núcleo familiar, pues también se constituye como el principal proveedor, como el sostén económico de la familia.

Los roles de género en América Latina y en Ecuador, son el resultado de construcciones sociales, 
políticas, religiosas que vinculan o que giran principalmente en torno a la familia. El proceso de feminización de la migración, ha generado una ruptura en algunos casos de estos estereotipos tradicionales de los roles masculino y femenino, mientras que en otros casos se ha ratificado y subrayado dichos roles. Al ser la mujer el primer eslabón en las cadenas migratorias, se produce un cambio en la idea de concebir al varón como el viajero y el aventurero, quien se arriesgaba en la odisea para alcanzar el 'sueño americano' para toda la familia que lo esperaba en el país de origen. "La salida de las mujeres no sólo ha producido un reacomodamiento en las relaciones de género y generacionales al interior de su grupo doméstico, sino que ha confrontado a la sociedad ecuatoriana con las transformaciones estructurales familiares, sociales y culturales producto de la denominada estampida de la población ecuatoriana" (Pedone, 2008).

Sin embargo, los cambios y transformaciones que surgen al interior de las familias ecuatorianas, en el contexto de la migración femenina, son un tanto problemáticas e incluso ambiguas, porque por un lado la mujer está ganando un peso mayor en los procesos de toma de decisiones dentro del familia, modificando los estereotipos de rol de género, pero a la par se reafirman algunas de las prácticas que tradicionalmente son asignadas a la mujer en la sociedad, tales como el cuidado de niños y ancianos, el servicio doméstico y la reproducción social. ${ }^{1}$ Es decir, que "las trayectorias migratorias de las mujeres que se insertan en las cadenas globales del cuidado, son un campo de análisis privilegiado para mirar cómo las desigualdades sociales y las relaciones de poder, que tejen el entramado social en origen, se traducen en el espacio transnacional" (Herrera, 2005).

Dentro de las familias que han iniciado procesos migratorios, es decir familias transnacionales, la reducción en el número de miembros conlleva a la redefinición de roles, deberes y obligaciones dentro del núcleo familiar, con base en las mismas características que eran desempeñadas por quienes han partido y por quienes se quedaron (Lagomarsino, 2005). Cuan-

1 "La reproducción de las relaciones sociales es la reproducción de determinado modo de vida, de lo cotidiano, de valores, de prácticas culturales y políticas y del modo como se producen las ideas en la sociedad. Ideas que se expresan en prácticas sociales, políticas, culturales, y en patrones de comportamiento y que acaban por permear toda la trama de relaciones de la sociedad." (Yazbek, 1998) do un esposo decide migrar, es la mujer quien generalmente asume ciertos roles que desempeñaba el padre dentro del núcleo familiar; sin embargo, cuando es la esposa quien migra, los padres frecuentemente dan un paso atrás y buscan que familiares mujeres se hagan cargo en parte de la crianza de hijos e hijas. Por lo tanto, al momento de migrar aquellas mujeres que también son madres, deben negociar con otras mujeres que se quedan para asegurar las redes de cuidado de hijos, hijas y ancianos en sus países de origen; es así que madres, abuelas, tías, hermanas, suegras pasan a ser ejes fundamentales para apoyar los proyectos migratorios encabezados por mujeres-madres. Cabe recalcar, que estas negociaciones se hicieron en un inicio pensando que el tiempo que estarían fuera sería relativamente corto, pero una vez que aquellas mujeres-madres se dieron cuenta de que su estancia en los países de destino sería más larga, debieron reacomodar los tratos hechos previamente, e incluso muchas optaron por plantear estrategias que tenían como objetivo la reunificación familiar en los nuevos países de residencia (Gündüz, 2013). Pero hasta que las estrategias de reunificación familiar se pongan en práctica, las mujeres-madres se ven obligadas a ejercer una maternidad transnacional, ya que crean relaciones sociales transfronterizas que requieren de nuevas estrategias que las ayuden a seguir ejerciendo el rol de madres a distancia.

Se calcula que para el año 1999 el $60 \%$ de los ecuatorianos y las ecuatorianas que migraron dejaron a hijas e hijos menores de edad en nuestro país, mientras que el 2001 se calculaba que el 5\% de la población total de niñas, niños y adolescentes tenían a sus padres y/o madres fuera del país (Pedone, 2008). Dadas las impresionantes cifras, y con la evidente feminización de la migración hacia España, es cuando se dispara la alarma y los discursos sociales, políticos, mediáticos y educativos se centran, específicamente, en la desintegración familiar, el abandono de los hijos e hijas, y la consiguiente estigmatización sobre el desempeño escolar y social de niños, niñas y adolescentes involucrados en estos contextos migratorios transnacionales (Pedone, 2008). A pesar de que muchos han estigmatizado a la mujer-madre migrante defendiendo un discurso de abandono y de desintegración familiar, es preciso reflexionar sobre dos puntos fundamentales. Primero, la migración de mujeres-madres ecuatorianas ha permitido que se visibilice la importancia de su rol de cuidadora dentro de las familias, el mismo 
que al estar naturalizado parecía no ser reconocido. Por otro lado, la migración de mujeres-madres trastoca los roles tradicionales de género, pues pasan a evidenciarse también como proveedoras. De hecho, "su rol de madres es el que está justificando la migración, pues se trata de cumplir con la responsabilidad de garantizar la reproducción social. Por ello es tan importante que su rol como proveedoras quede claro y legitimado. Proveedoras y cuidadoras no son dos funciones separadas, más bien son percibidas como íntimamente articuladas" (Herrera, 2013).

\section{Conclusiones}

La feminización de la migración ecuatoriana ha significado la reconfiguración de los imaginarios de género, así como de las dinámicas sociales y familiares en nuestro país. Hemos podido analizar cómo la ola migratoria de 1998-2001, llevó a miles de mujeres a iniciar una travesía transatlántica, debido a la crisis económica, política y social que vivía el Ecuador. La gran mayoría de ellas iniciaría sus actividades laborales en los países de destino, tales como España e Italia, en el sector del cuidado de niños y ancianos. Este hecho resulta chocante y tremendamente irónico, ya que en el caso de las madres migrantes, en muchas ocasiones, tuvieron que dejar a sus propios hijos, cruzar un océano y dedicarse a cuidar a hijos ajenos. La figura de la mujer migrante, ha sido criticada y estigmatizada, debido al reproche de la sociedad con respecto a dejar solos a sus hijos o hijas; y en otros casos, también son censuradas, por habérselos llevado consigo, a vivir en condiciones precarias. La maternidad transnacional supone un nuevo reto, pues las madres deben negociar la puesta en marcha de nuevas redes de cuidado en el país de origen con el apoyo de abuelas, tías y hermanas. Las ecuatorianas madres y migrantes logran articular dos funciones fundamentales que marcarán en gran medida, la redefinición de su papel dentro de la familia, pues convergen su rol de cuidadora y proveedora bajo el contexto migratorio. Por lo tanto, es preciso pensar en la familia migrante como un nuevo actor en la sociedad, que dista del ideal de familia que se presume que existe, pues tiene lugar una reconfiguración de las dinámicas familiares dada la situación transnacional de la que pasan a formar parte.

\section{Referencias}

Bastia, Tanja. (2008). La feminización de la migra- ción transnacional y su potencial emancipador. Revista Papeles de Relaciones Ecosociales y cambio global, (104), 67-77. Recuperado desde http://www.fuhem.es/media/ecosocial/ file/Democracia/Diversidad\%20y\%20migraciones $/ \mathrm{la} \% 20$ feminizacion $\% 20 \mathrm{de} \% 201 \mathrm{a} \% 20$ pobreza_T.BASTIA.pdf

Genta, N., \& Ramírez, J. (2008). ECUADOR: La migración internacional en cifras. FLACSO-Ecuador y Fondo de Población de las Naciones Unidas UNFPA. Recuperado desde http:// www.flacsoandes.edu.ec/libros/digital/43598. pdf

Gündüz, Zuhal. (2013). The Feminization of Migration. Monthly Review, 65 (7). Recuperado desde http://monthlyreview.org/2013/12/01/ feminization-migration/\#top

Herrera, G., et al. (2005). Mujeres ecuatorianas en redes globales del cuidado. La migración ecuatoriana transnacionalismo, redes e identidades. FLACSO-Ecuador y Plan Migración, Comunicación y Desarrollo Fepp. Recuperado desde http://www.flacsoandes.edu.ec/web/ imagesFTP/6417.migracion_ecuatoriana transnacionalismo_redes_e_identidades.pdf

Herrera, G. (2013). Migración, cuidados y familias transnacionales. "Lejos de tus pupilas" Familias transnacionales, cuidados y desigualdad social en Ecuador. Recuperado de http:// www.flacsoandes.edu.ec/libros/digital/54078. pdf

Lagomarsino, F., et al. (2005). ¿Cuál es la relación entre familia y migración? El caso de las familias de emigrantes ecuatorianos en Génova. La migración ecuatoriana: transnacionalismo, redes e identidades. FLACSO-Ecuador y Plan Migración, Comunicación y Desarrollo Fepp. Recuperado desde http://www. flacsoandes.edu.ec/web/imagesFTP/6417.migracion_ecuatoriana_transnacionalismo_redes_e_identidades.pdf

Pedone, Claudia. (2008). Varones aventureros vs. Madres que abandonan: reconstrucción de las relaciones familiares a partir de la migración ecuatoriana. Revista Interdisciplinaria da Mo- 
bilidade Humana REMHU, (30), 45-64. Recuperado desde https://giim.files.wordpress. com/2008/11/remhu-claudia-pedone.pdf

Pedone, C. (2010). Las migraciones latinoamericanas en Cataluña. Generalitat de Catalunya Departament d'Acció Social i Ciudadania. Recuperado desde https://www.youtube.com/ watch? $\mathrm{v}=\mathrm{a} 9$ pafg7bH5I

Penson, C. (2007). Feminization of Migration. Gender, Remittances and Development INSTRAW, working paper 1. Recuperado desde http://www.renate-europe.net/wp-content/ uploads/2014/01/Feminization_of_Migration-INSTRAW2007.pdf

Torres, F. (2004). La migración ecuatoriana en España y su proceso de inserción. Revista Aportes Andinos Programa Andino de Derechos Humanos. Recuperado desde http://www.uasb. edu.ec/padh/revista12/migracion/ponencias/ francisco $\% 20$ torres.htm

Unda, R., \& Alvarado, S. (2012). Feminización de la migración y papel de las mujeres en el hecho migratorio. Revista Latinoamericana de Ciencias Sociales, Niñez y Juventud, 10 (1), 593610. Recuperado desde http://www.scielo. org.co/pdf/rlcs/v10n1/v10n1a 